\title{
Domination masculine et représentations sexuées des acteurs du champ politique : une approche par les lapsus d'adresse
}

Masculine domination and sexual representations of political field actors: an approach by mistitlings

Dominación masculina y representaciones del sexo de agentes del campo político: un análisis vía los lapsus de la denominación

\section{David Descamps et Agathe Foudi}

\section{OpenEdition \\ Journals}

Édition électronique

URL : https://journals.openedition.org/mots/23603

DOI : $10.4000 /$ mots. 23603

ISSN : 1960-6001

\section{Éditeur}

ENS Éditions

\section{Édition imprimée}

Date de publication : 5 novembre 2018

Pagination : 139-154

ISBN : 979-10-362-0082-3

ISSN : 0243-6450

\section{Référence électronique}

David Descamps et Agathe Foudi, « Domination masculine et représentations sexuées des acteurs du champ politique : une approche par les lapsus d'adresse », Mots. Les langages du politique [En ligne], 118 | 2018, mis en ligne le 05 novembre 2020, consulté le 23 avril 2022. URL : http:// journals.openedition.org/mots/23603; DOI : https://doi.org/10.4000/mots.23603 


\section{Domination masculine et représentations sexuées des acteurs du champ politique : une approche par les lapsus d'adresse}

L'étude des représentations genrées et sexuées ${ }^{1}$ que les acteurs du champ politique (personnalités politiques ou commentateurs professionnels) peuvent avoir des différents éléments qui composent leur champ (ses fonctions, ses statuts, ses participants, etc.) pose un problème d'ordre méthodologique. Comme ces acteurs peuvent contrôler leur discours, celui-ci ne garantit pas d'accéder à leurs représentations dès lors qu'il s'opère sur un mode volontaire, ces acteurs ayant en effet la possibilité d'instrumentaliser leur propos pour renvoyer une bonne image d'eux-mêmes ${ }^{2}$. Et, comme les acteurs du champ politique ne sont pas pleinement conscients de leurs représentations psychiques, on peut aussi penser qu'ils ne pourraient que difficilement en rendre compte3.

Parce qu'il s'agit d'actes commis involontairement, les lapsus 4 semblent en revanche constituer un instrument ouvrant la voie à une analyse efficace de ces représentations.

1. Nous parlerons de représentations à propos de la manière dont les catégories sociopsychiques des individus s'agencent contextuellement et circonstanciellement pour apprécier la réalité, de représentations sexuées à propos de celles liées directement au sexe des individus («monsieur», «madame», etc.) et de représentations genrées à propos de celles qui lui sont liées indirectement et peuvent donc s'appliquer à des choses qui débordent les seuls individus («masculinité » ou «féminité » d'un métier, d'une activité, etc.).

2. Comme le relevait Erving Goffman, dans le cadre de la mise en scène de sa vie quotidienne, « un acteur peut toujours transmettre intentionnellement de fausses informations» (Goffman, 1973, p.12).

3. On rejoint cette fois, au moins partiellement, le principe sociologique consistant à considérer que les individus ne connaissent pas forcément « les véritables raisons qui [les] ont déterminé[s] à agir et la nature de [leur] action», qu'ils «sav[ent] bien mal les mobiles relativement simples qui [les] guident» (Durkheim, 2005 [1901], p.xIV).

4. Nous nous référons ici à la définition dictionnairique du mot «lapsus», à savoir la substitution involontaire d'un mot ou d'un groupe de mots par un autre dans un propos. Nous nous écartons donc de la définition linguistique française qui associe ce mot à ce qui est désigné en anglais par le «slip of the tongue» - littéralement «glissement de la langue» - ou en allemand par le «versprechen» - littéralement «mal-dire». Notre objet renvoie à ce que Pierre Arnaud appelle «lapsus par substitution de mots» (Arnaud, 1997).

Université de Lille, Clersé (UMR 8019)

daviddescamps@hotmail.com

Université de Lille, Clersé (UMR 8019)

agathe.foudi@hotmail.fr 
Même si les lapsus ont surtout été étudiés dans une perspective psychanalytique ou linguistique - le phénomène étant appréhendé dans le premier cas comme formation de compromis entre processus psychiques conscients et inconscients (Freud, 2001 [1922]) ; dans le second comme un instrument permettant de développer des modèles de production langagière (Fromkin, 1971) - on ne peut exclure a priori la pertinence d'une analyse sociologique du phénomène. Substitutions involontaires d'un mot ou d'un groupe de mots par un autre dans un propos, les lapsus linguae mettent en effet toujours en jeu des connaissances et ne peuvent donc être que le produit, au moins partiel, de la trajectoire sociohistorique de ceux qui les commettent. À ce titre, ils peuvent constituer un objet sociologique dans la tradition désormais constituée de la sociologie de la connaissance, voire un instrument permettant de saisir la connaissance des individus et les représentations qui en découlent.

Si l'on considère en effet que les représentations des individus reposent sur l'existence de structures structurées de relations et de rapports catégoriels5, ces représentations devraient pouvoir s'observer au travers même des lapsus qui, par définition, mettent toujours en jeu au moins une catégorie substitut et au moins une catégorie substituée. Par ailleurs, comme l'une s'impose aux dépens de l'autre, on peut chercher à observer les relations, voire les rapports, qui unissent ces catégories (Bourdieu, 1984) ${ }^{6}$; l'idée étant que la substitution d'une catégorie à une autre peut reposer non seulement sur des similitudes catégorielles, mais aussi sur des différentiels de force d'imposition7. Enfin, comme les lapsus ont un caractère involontaire, on peut penser que les relations qui s'observent quand ils sont commis traduisent davantage les représentations réelles de leurs auteurs (c.-à-d. les représentations psychiques dont ils disposent effectivement) que ne pourrait le faire un propos formulé sur le mode du volontaire.

Dans la mesure où il s'agit d'étudier ici les représentations sexuées et genrées des acteurs du champ politique, l’idée qui nous a guidés a été de considérer que l'analyse de ces lapsus, au travers desquels Muguette Jacquaint a pu, par exemple, se faire appeler du patronyme de Pierre Juquin (1), ou Catherine

5. On parlera de relations catégorielles dans un sens large pour désigner les liens qui peuvent s'établir entre catégories sociopsychiques, de relations catégorielles dans un sens restreint à propos de liens qui ne sont pas hiérarchisés, et de rapports catégoriels à propos de liens qui le sont.

6. Pierre Bourdieu indique que «[s]i les rapports de force objectifs tendent à se reproduire dans les visions du monde social qui contribuent à la permanence de ces rapports, c'est donc que les principes structurants de la vision du monde prennent leur racine dans les structures objectives du monde social et que les rapports de force sont aussi présents dans les consciences [...] » (Bourdieu, 1984, p. 6).

7. Nous pensons que s'il existe des représentations mentales dominantes et des représentations mentales dominées, alors les catégories qui les fondent peuvent elles-mêmes être dominantes ou dominées et disposer pour cette raison d'une capacité d'imposition dans l'énonciation inégale suivant les rapports de domination qui structurent la «vie réelle» (Marx, Engels, 1977 [1845], p. 50). 
Tasca se faire appeler «monsieur» (2), pouvait être riche d'enseignements sur les représentations des acteurs et des actrices du champ politique.

(1) M. le ministre des affaires sociales et de l'emploi [Philippe Séguin]. [...] monsieur Collomb, je me félicite de votre évolution et de vous entendre trouver des vertus à mon texte [...]. Mais, vous le savez, je suis de ceux qui n'ont pas nié qu'il y avait eu parfois une évolution dans vos rangs depuis 1981! (Sourires.) Mme Jucquaint a évoqué...

Mme Muguette Jacquaint. Mme Jacquaint ! (Rires.)

(JO-AN, $2^{\mathrm{e}}$ séance du vendredi 25 avril 1986, p. 424-425)

(2) M. Jean-Louis Debré. [...] Or, monsieur le ministre, monsieur le président de la commission des lois [...]

M. Gérard Gouzes, rapporteur. C'est une présidente! Lapsus révélateur!

M. Jean-Louis Debré. Madame la présidente de la commission, voulais-je dire.

(JO-AN, $1^{\text {re }}$ séance du jeudi 11 décembre 1997, p. 10)

Ces lapsus qui se traduisent par la substitution de prénoms et/ou de noms de personnalités politiques (formes d'adresse personnelle) et ceux qui mettent en jeu les catégories langagières de "madame » ou de "monsieur ${ }^{8}$ (formes d'adresse de civilité) présentent en effet un intérêt patent pour saisir les représentations sexuées et genrées de leurs auteurs : comme les individus mis en cause ont des propriétés statutaires et sexuelles objectivables, on peut analyser par leur biais des relations psychiques entre des catégories marquées par le genre ou le sexe.

Pour constituer notre corpus (267 cas de lapsus d'adresse personnelle et 12 cas de lapsus d'adresse de civilité), nous avons eu recours aux moteurs de recherche associés aux bases archivant des journaux de la presse (officielle ou non) ou d'autres documents rapportant des propos tenus par des acteurs politiques9. Puis, de manière générale, nous avons formulé des requêtes à partir de mots-clés souvent utilisés lorsque des lapsus sont commis («lapsus», "pardon », "voulais-je dire », « excusez-moi », etc.). Si nous ne pouvons être certains que tous les cas qui composent notre corpus soient de vrais lapsus - ceux-ci pouvant être feints -, les "faux» lapsus y sont probablement peu nombreux dans la mesure où les substitutions en question se révèlent dans le champ politique souvent «coûteuses» pour leurs auteurs. Bien évidemment, le corpus à partir duquel il s'agit d'entreprendre notre analyse ne peut prétendre ni à la complétude ni même à une parfaite représentativité des lapsus produits. En dépit

8. Les expressions que nous avons adoptées sont inspirées de l'ouvrage dirigé par Catherine Kerbrat-Orecchioni qui s'intitule S'adresser à autrui : les formes nominales d'adresse en français. Dans l'introduction, C. Kerbrat-Orecchioni écrit que "[l]es formes monsieur/madame/mademoiselle [...] sont les termes d'adresse "passe-partout” [...]». Une note associée à ce passage précise : «On parle parfois à ce sujet de “(forme de) civilité” » (Kerbrat-Orecchioni éd., 2010, p. 20).

9. Il s'agit essentiellement des bases constituées par l’Assemblée nationale, le Sénat, la Bibliothèque nationale de France (gallica.bnf.fr), l'Institut national de l'audiovisuel, la Direction de l'information légale et administrative (vie-publique.fr), ainsi que de la base Europresse. 
de notre souci d'exhaustivité, une partie conséquente des lapsus commis par les acteurs du champ politique a pu nous échapper, soit qu'elle n'ait pas été repérée par nos soins, soit qu'elle n'ait pas été enregistrée dans les bases que nous avons consultées ${ }^{10}$. Quoi qu'il en soit, même si le nombre de lapsus réunis ne représente qu'une part faible de l'ensemble des lapsus d'adresse qui ont été produits dans le champ politique sur la période considérée (du début de la IIIe République à la fin de l'année 2016), la taille de notre échantillon est assez importante pour mettre au jour des régularités permettant d'observer les représentations sexuées et genrées des acteurs du champ politique et leur évolution.

\section{Un champ masculin}

Nos données montrent d'emblée que la mise en cause des individus au travers de lapsus d'adresse personnelle dépend de leur sexe et de la place respective qui a été attribuée dans le champ politique aux hommes et aux femmes. Ainsi, jusqu'en 1944, nous n'avons pas pu enregistrer de lapsus mettant en cause des personnalités féminines. L'absence manifeste de tels lapsus sur un corpus somme toute restreint $(n=11)$ peut s'expliquer par une production relativement rare, en lien avec l'exclusion objective des femmes du champ politique et les représentations sociales sexuellement différenciées dont pouvaient disposer les acteurs du champ ${ }^{11}$.

Si l'obtention par les femmes du droit de vote et d'éligibilité en 1944 et la consécration de l'égalité civile et juridique entre hommes et femmes (Constitution du 27 octobre 1946) ont offert aux femmes une place en politique, celleci est restée marginale jusqu'à la première loi sur la parité (Sineau, 2011) ${ }^{12}$. La domination masculine, qui peut alors se lire objectivement au travers de la faible place dévolue aux femmes, s’observe également au travers des représentations des acteurs du champ politique que nos cas de lapsus permettent de saisir. La structure des cas qui couvrent cette période révèle en effet que, dans leurs représentations, la masculinité est alors étroitement associée à ce champ et à ses statuts (voir tableau 1).

10. Certains lapsus ont pu ne pas être repérés, quand d'autres ont pu disparaître des comptes rendus (officiels ou non) parce que les rédacteurs de journaux n'ont pas jugé opportun d'en rendre compte ou parce qu'ils ont été amenés à les effacer volontairement, notamment dans les Journaux officiels (Coniez, 2008).

11. Dans les représentations essentialisantes des femmes qui gouvernent les acteurs du champ sous la IIIe République, nul n'est plus éloigné de la sphère publique que ne l'est « la» femme. On trouve chez le républicain Eugène Pelletan l'idée que la place de «la » femme est « au foyer » : «Le mari ministre des affaires étrangères, la femme ministre de l'intérieur, et toutes les questions du ménage décidées en conseil des ministres» (E. Pelletan, 1869, "La femme au xıx siècle», La revue politique et littéraire : revue des cours littéraires, vol. II, $\mathrm{n}^{\circ}{ }_{15}$, p. 235-240, p. 239).

12. Mariette Sineau parle, à propos de la période 1958-1995, de «hors-jeu politique des femmes » (Sineau, 2011, p. 19). 
Tableau 1. Répartition réelle et écart à une répartition théorique des lapsus commis par des acteurs du champ politique et mettant en cause au moins un élément de l'adresse personnelle de personnalités politiques (1944-1999)

\begin{tabular}{|c|c|c|c|c|c|c|c|}
\hline \multirow{3}{*}{$N=37$} & & \multicolumn{4}{|c|}{$\begin{array}{c}\text { Sexe de l'individu au prénom et/ou } \\
\text { au nom substituts }\end{array}$} & & \\
\hline & & \multicolumn{2}{|c|}{ Homme } & \multicolumn{2}{|c|}{ Femme } & \multicolumn{2}{|c|}{ Total } \\
\hline & & $\begin{array}{l}\text { Réel } \\
\text { (en \%) }\end{array}$ & $\begin{array}{l}\mathrm{El}^{\mathrm{a}} \text { (en } \\
\text { points) }\end{array}$ & $\begin{array}{c}\text { Réel } \\
\text { (en \%) }\end{array}$ & $\begin{array}{c}\text { El (en } \\
\text { points) }\end{array}$ & $\begin{array}{l}\text { Réel } \\
\text { (en \%) }\end{array}$ & $\begin{array}{l}\text { El (en } \\
\text { points) }\end{array}$ \\
\hline \multirow{3}{*}{$\begin{array}{l}\text { Sexe } \\
\text { de l'individu } \\
\text { au prénom et/ } \\
\text { ou au nom } \\
\text { substitués }\end{array}$} & Homme & 84 & -1 & 0 & -7 & 84 & -8 \\
\hline & Femme & $\underline{16}$ & $\underline{9}$ & 0 & -1 & 16 & 8 \\
\hline & Total & 100 & 8 & 0 & -8 & 100 & 0 \\
\hline
\end{tabular}

a. El = écart à l'indépendance

Source : base de données personnelle.

Lecture (données soulignées) : entre 1944 et 1999, 16 \% des lapsus de notre corpus (commis par des acteurs du champ politique à l'égard d'au moins un individu du champ politique) se sont traduits par l'octroi d'un prénom et/ou d'un nom «substituts masculins» à un individu dont le prénom et/ou le nom «substitués» correspondent à celui d'une femme. C'est 9 points de plus que ce qui aurait prévalu dans le cadre d'une formation et d'une distribution des lapsus d'adresse personnelle réalisées indépendamment du sexe des individus mis en cause.

La domination masculine que connaît le champ politique y est si puissante qu'elle autorise en fait très largement la transgression de l'identité sexuelle des individus de sexe féminin : parmi les 37 cas de lapsus que nous avons relevés sur la période, tous se sont traduits par l'attribution du prénom et/ou du nom d'un homme en lieu et place de celui/ceux d'un autre individu, que celui-ci ait été un homme (31 cas) ou une femme ( 6 cas). Contrairement à ce qui aurait prévalu dans le cadre d'une distribution réalisée indépendamment du sexe des individus mis en cause, on observe ainsi une très nette surreprésentation des lapsus commis à l'égard de femmes qui se sont traduits par l'imposition de l'adresse personnelle d'hommes, et une très nette sous-représentation des lapsus commis à l'égard d'hommes et qui auraient dû se traduire par l'imposition de l'adresse personnelle de femmes. La remarque de Véronique Neiertz en 1997 - «Déjà qu'il n'y a pas beaucoup de femmes dans cette assemblée »-à l'intention de Jacques Myard qui l'avait appelée «monsieur Gremetz » (3) reflète finalement très bien ce que le phénomène des lapsus d'adresse traduit dans la seconde moitié du $x x^{e}$ siècle : c'est bien la visibilité de l'identité des individus de sexe féminin qui est remise en cause lorsque de tels lapsus sont commis par les acteurs du champ. 
(3) Mme Véronique Neiertz. Et la sanction du travail clandestin, alors?

M. Jacques Myard. Ce texte promet de le poursuivre, M. Gremetz! (Rires), pardon, madame Neiertz! [...]

Mme Véronique Neiertz. Déjà qu'il n'y a pas beaucoup de femmes dans cette assemblée [...]

(JO-AN, $2^{\mathrm{e}}$ séance du jeudi 20 février 1997, p. 36)

Mais les lapsus d'adresse commis à l'égard des femmes en leur appliquant un prénom et/ou un nom renvoyant à un individu de sexe masculin ne constituent pas seulement une transgression de leur identité sexuelle. Parce que les statuts, le personnel et le champ politique sont conçus et pensés comme masculins, de tels lapsus traduisent aussi le respect d'une représentation genrée dans laquelle le masculin domine.

Mettant en cause des individus des deux sexes, les lapsus d'adresse personnelle réalisés entre 1944 et 2000 sont donc finalement assez révélateurs du fait que le champ politique était alors encore très fortement marqué du point de vue des représentations par la domination masculine.

\section{Un champ qui s'ouvre au genre féminin}

Si, à la veille du xxI e siècle, les femmes sont encore largement sous-représentées dans le champ politique, les lois sur la parité vont conduire à un processus incontestable de féminisation du personnel du champ. Sans surprise, la part des lapsus mettant en cause des femmes s'est alors accrue; et ce à raison de la place que ces dernières y ont occupée.

Du point de vue de notre objet, ce sont en fait les mutations formelles des lapsus d'adresse qui sont intéressantes. La structure des cas de lapsus de notre corpus montre en effet que, sur cette période, le sexe des individus apparaît comme suffisamment saillant dans les représentations pour que son respect soit un phénomène dominant (voir tableau 2). Autrement dit, lorsque des lapsus sont commis, les acteurs du champ politique apparaissent désormais suffisamment sensibles au sexe de celles et ceux dont ils souhaitent parler pour ne pas le transgresser. 
Tableau 2. Répartition réelle et écart à une répartition théorique des lapsus commis par des acteurs du champ politique et mettant en cause au moins un élément de l'adresse personnelle de personnalités politiques (2000-2016)

\begin{tabular}{|c|c|c|c|c|c|c|c|}
\hline \multirow{3}{*}{$N=219$} & & \multicolumn{4}{|c|}{$\begin{array}{l}\text { Sexe de l'individu au prénom et/ou } \\
\text { au nom substituts }\end{array}$} & \multirow{2}{*}{\multicolumn{2}{|c|}{ Total }} \\
\hline & & \multicolumn{2}{|c|}{ Homme } & \multicolumn{2}{|c|}{ Femme } & & \\
\hline & & $\begin{array}{l}\text { Réel } \\
\text { (en \%) }\end{array}$ & $\begin{array}{l}\text { Ela (en } \\
\text { points) }\end{array}$ & $\begin{array}{l}\text { Réel } \\
\text { (en \%) }\end{array}$ & $\begin{array}{c}\text { El (en } \\
\text { points) }\end{array}$ & $\begin{array}{l}\text { Réel } \\
\text { (en \%) }\end{array}$ & $\begin{array}{l}\text { El (en } \\
\text { points) }\end{array}$ \\
\hline \multirow{3}{*}{$\begin{array}{l}\text { Sexe de l'individu } \\
\text { au prénom et/ } \\
\text { ou au nom } \\
\text { substitués }\end{array}$} & Homme & 79 & 8 & 3 & -10 & 82 & -2 \\
\hline & Femme & $\underline{8}$ & $\underline{-6}$ & 11 & 8 & 18 & 2 \\
\hline & Total & 86 & 2 & 14 & -2 & 100 & 0 \\
\hline
\end{tabular}

a. El = écart à l'indépendance

Source : base de données personnelle.

Lecture (données soulignées) : entre 2000 et 2016, 8 \% des lapsus de notre corpus se sont traduits par l'octroi d'un prénom et/ou d'un nom «substituts masculins» à un individu dont le prénom et/ou le nom «substitués» correspondent à celui d'une femme. C'est 6 points de moins que ce qui aurait prévalu dans le cadre d'une formation et d'une distribution des lapsus d'adresse personnelle réalisées indépendamment du sexe des individus mis en cause.

Parmi les 219 cas de lapsus que nous avons relevés sur la période, 172 ont été réalisés à l'égard d'hommes qui se sont vu attribuer le prénom et/ou le nom d'un autre homme, et seulement 7 à l'égard d'hommes qui se sont vu attribuer le prénom et/ou le nom d'une femme. Quant aux femmes, 17 se sont vu attribuer le prénom et/ou le nom d'un homme et 23 celui d'une femme. Par rapport à une situation théorique où les lapsus se seraient distribués indépendamment de la variable sexe ${ }^{13}$, les femmes ont ainsi été plus confondues qu'elles n'auraient dû l'être avec d'autres femmes et les hommes avec d'autres hommes ${ }^{14}$. Alors qu'au xxe siècle, la production de ce type de lapsus semblait très improbable, au $x x^{e}$, Nathalie "Kosciusko-Morizet» peut aisément être confondue avec Anne «Hidalgo » (4), tout comme « Marine Le Pen» avec «Martine Aubry» (5).

13. Nous parlons ici et dans les tableaux 1 et 2 de répartition théorique à propos d'une distribution de lapsus qui serait indépendante du sexe des individus mis en cause. Cette répartition a été calculée en fonction de la probabilité associée à deux tirages aléatoires respectant la proportion qu'hommes et femmes représentent parmi l'ensemble des individus en cause dans les lapsus de notre corpus, tirages visant à désigner respectivement le sexe de l'individu dont le prénom et/ou le nom se sont effacés et le sexe de celui dont le prénom et/ou le nom se sont imposés.

14. Alors qu'un individu (homme ou femme) aurait dû, de manière théorique, être 5,3 fois plus confondu avec un autre homme qu'avec une femme, les hommes l'ont été 24,6 fois; les femmes ayant quant à elles été 1,3 fois plus confondues avec une autre femme qu'avec un homme. 
(4) Mme Nathalie Kosciusko-Morizet. [...] Chers collègues, notre Assemblée n'a pas à écarter quelqu'un ad personam [...]

M. Olivier Dussopt, rapporteur. Comme vient de le dire Mme Hidalgo... (Rires.) Veuillez m'excuser, madame la députée. Comme vient de le dire Mme KosciuskoMorizet [...].

(JO-AN, $3^{\text {e }}$ séance du jeudi 2 juillet 2015, p. 6436)

(5) Vincent Peillon. Il y a au premier tour des candidats du Front de gauche, du Parti socialiste [...]. Et puis il y aura un second tour. J'espère d'ailleurs que Martine Aubry n'y sera pas... Martine Aubry, euh pardon, Marine Le Pen n'y sera pas.

(Questions d'Info, LCP-AN, 23 mai 2012)

On peut voir dans ce phénomène un recul significatif de l'assimilation pure et simple de l'exercice du pouvoir politique à la masculinité sans que l'on puisse toutefois en déduire la disparition de la domination masculine. En effet, si, quel que soit leur sexe, les individus ont été plus souvent confondus à l'occasion de lapsus avec des individus de même sexe qu'avec d'autres, on observe aussi que la distribution de ces lapsus ne s'est pas opérée indépendamment du sexe des individus mis en cause. Tandis que les prénoms et/ou noms féminins sont surreprésentés parmi les prénoms et/ou noms substitués, ceux des hommes le sont parmi les prénoms et/ou noms substituts (voir tableau 2). Autrement dit, "Martine Aubry » ou «Michèle Alliot-Marie» semblent pouvoir être plus facilement appelées respectivement «Martine Hollande» (6) et «François Baroin » (7) que «François Hollande » et «François Baroin » ne peuvent être appelés «François Aubry» et « Michèle Alliot-Marie».

(6) Claude Guéant. Écoutez, moi je ne connais pas François Hollande autrement que par les médias, par ses déclarations et je pense qu'il est intéressant de se pencher sur les appréciations que portent sur lui ceux qui le connaissent. [...] Et ceux-ci sont particulièrement sévères, enfin je me souviens de Martine Hollande qui...

Christophe Barbier. Martine Aubry.

Claude Guéant. Martine Aubry, excusez le lapsus significatif [...]

(I-Télé, 17 octobre 2011)

(7) M. Brice Hortefeux, ministre de l'intérieur. [...] il n'y a pas si longtemps que j'ai pris mes fonctions, succédant à François Baroin (Rires), pardon à Michèle AlliotMarie qui lui a elle-même succédé! [...]

(JO-AN, $1^{\text {re }}$ séance du mercredi 10 février 2010, p. 1033)

Par rapport à une situation où il y aurait une distribution indépendante du sexe des individus, on voit donc qu'il existe une sous-représentation bien plus forte des cas où le prénom et/ou le nom d'un homme s'effacent au profit de celui (ou de ceux) d'une femme (10 points) que des cas où le prénom et/ ou le nom d'une femme s'effacent au profit de celui (ou de ceux) d'un homme (6 points). 
Si, par la force de reconnaissance dont bénéficient désormais les identités sexuelles dans le champ politique, les lapsus ne se traduisent plus aussi systématiquement par la disparition des adresses personnelles des individus de sexe féminin, la domination masculine perdure bel et bien dans les représentations puisque à ce phénomène se combine donc toujours une prime au masculin.

\section{Sexe, genre et prestige des fonctions politiques}

Comme en ce qui concerne les lapsus d'adresse personnelle, on observe que le féminin tend à s'effacer davantage que le masculin dans le cadre des lapsus portant sur les adresses de civilité. Tandis que les hommes politiques sont assez rarement victimes de transgressions de leur forme de civilité, de nombreux lapsus témoignent en effet d'une tendance à substituer au nom commun de «Madame » celui de «Monsieur», notamment lorsque les femmes politiques désignées occupent ou briguent des fonctions prestigieuses ${ }^{15}$. Si le lapsus commis par Jean Auroux à l'égard de la Première ministre Édith Cresson en l'appelant «Monsieur» est passé à la postérité (8), bien d'autres ont été commis et relevés depuis que des femmes ont acquis des statuts prestigieux dans le champ. Mais il est intéressant d'observer que, tandis que la masculinisation des formes d'adresse de civilité des femmes est souvent opérée dans le cadre de propos faisant explicitement référence à un statut ou à une fonction politique valorisés (9), on tend plutôt à féminiser celui des hommes lorsqu'on fait abstraction de leur statut ou de leur fonction politique (10).

(8) Jean Auroux. Monsieur le Premier ministre... Madame... C'est la première fois depuis si longtemps...

(Journal de 20 heures, Antenne 2, 31 mai 1991)

(9) Mme la présidente [Sandrine Mazetier]. La parole est à M. le ministre, pour donner l'avis du Gouvernement. (Exclamations.) [...]

Mme la présidente. «Mme la ministre», voulais-je dire... Excusez-moi !

Mme Marylise Lebranchu, ministre. Ce n'est pas grave, monsieur le président ! (Rires.)

(JO-AN, $2^{\mathrm{e}}$ séance du 7 novembre 2014, p. 8540)

(10) Mme le ministre de l'emploi et de la solidarité [Martine Aubry]. J'ajoute que le Gouvernement fait tous les jours de la parité son objectif [...]. Ce matin encore, Mme Guigou vient de nommer une femme procureur général. [...]

Il y a peu de temps, Mme Allègre a désigné la première femme président [...] $M$. Allègre voulais-je dire!

(JO-AN, séance du 21 janvier 1998, p. 11-12)

15. On parlera de fonctions ou de statuts prestigieux à propos de ces statuts ou fonctions qui offrent à ceux qui les occupent un important «privilège positif [...] de considération sociale » (Weber, 1995 [1922], p. 395). 
La faible présence de lapsus mettant en cause l'adresse de civilité des hommes lorsque leur statut politique est mobilisé dans le propos tenu par les acteurs du champ peut s'expliquer par le fait qu'une telle mobilisation redouble symboliquement le caractère sexuellement masculin de celui qui le porte. Par contre, l'absence de référence au statut politique peut contribuer à faciliter l'émergence de tels lapsus en ce que, caractéristique du dominé, l'absence de statut politique entre en congruence avec l'attribut du féminin, attribut dominé dans le champ politique. Mutatis mutandis, si de nombreux lapsus affectant l'adresse de civilité des femmes sont produits alors qu'on ne fait référence qu'à leur statut, c'est certainement cette fois parce qu'en omettant dans le propos leur adresse personnelle (qui renvoie à un individu de sexe féminin) et en introduisant des statuts prestigieux, on tend à ne faire valoir que des éléments qui sont caractéristiques des dominants du champ et qui entrent en congruence avec l'attribut masculin.

Cela étant dit, à rebours de la croyance selon laquelle les femmes seraient toujours en situation défavorable dans le phénomène de domination masculine, ces lapsus invitent aussi à penser que certaines d'entre elles peuvent parfois en connaître le bénéfice symbolique. Parce qu'il existe une confusion hystérétique (Bourdieu, 1979, p. 122) du masculin et du prestige de la haute fonction politique, et parce qu'une femme politique peut cesser « d'être regardée comme femme» (Pionchon, Derville, 2004, p. 90) dès lors que ses «charmes » s'émoussent (Sineau, 1988, p. 48-50), la possession par certaines femmes de fonctions politiques prestigieuses fait qu'elles peuvent être perçues, catégorisées et qualifiées eu égard à leur statut et donc qu'on en vienne à leur attribuer un «titre » de civilité valorisé (masculin) inadéquat au regard de leur sexe (féminin). À ce titre, Natacha Bouchart a pu parler de la ministre de l'Intérieur (Home Secretary) britannique en face d'elle en l'appelant «Monsieur le ministre» alors même qu'il s'agissait de Theresa May (11).

(11) [...] Natacha Bouchart a dit «Monsieur le ministre» en parlant du ministre de l'Intérieur britannique, qui était assis en face d'elle. Et le ministre de l'Intérieur britannique s'appelle Theresa May, et c'est une dame.

("Dessous sans dentelles», La Voix du Nord, 8 juin 2011)

Cela étant, parce que la domination masculine passe aussi par une confusion entre le masculin et des comportements ou manières associés à l'exercice de ces fonctions, un certain nombre de lapsus apparaissent parfois liés au fait que les acteurs du champ politique peuvent lire la masculinité dans l'exercice que certaines femmes politiques font de leurs fonctions. C'est alors parce qu'elles adoptent des comportements et des manières perçus comme masculins que ces femmes peuvent être victimes de lapsus au travers desquels on masculinise leur adresse de civilité. Le lapsus réalisé par Lionel Jospin à l'égard de Roselyne Bachelot en octobre 2000 apparaît particulièrement symptomatique de ce type de cas (12). 
(12) Mme Roselyne Bachelot-Narquin. Monsieur le Premier ministre, le Président de la République s'est expliqué devant les Français, expliquant que la vidéo le mettant en cause relevait d'une manipulation et d'une opération mensongère pour ce qui le concerne. (Exclamations sur les bancs du groupe socialiste.) Les faits intervenus depuis le confirment.

Monsieur le Premier ministre, vous ne vous êtes pas encore expliqué directement devant les Français, alors que cette cassette vous met personnellement en cause en tant que premier secrétaire du Parti [...]

Le Président de la République s'est exprimé. Nous souhaitons qu'à votre tour vous nous apportiez les éclaircissements nécessaires. [...]

M. Lionel Jospin, Premier ministre. Monsieur le député, [excuses], j’ai du mal à comprendre la logique...

(VO-AN, $2^{\mathrm{e}}$ séance du 10 octobre 2000, p. 6588 ; «Le plein de questions, le vide de réponses », Liberation.fr, 11 octobre 2000) ${ }^{16}$

Commis alors que Lionel Jospin était interrogé dans le cadre de l'affaire Méry sur d'éventuelles sommes perçues par le PS, le lapsus peut en effet être mis en rapport avec l'attitude adoptée par Roselyne Bachelot. Alors qu'Élisabeth Guigou venait d'adopter une position politique «conciliante » à l'égard du président Jacques Chirac en rejetant l'idée d'un pourvoi dans le cadre de l'affaire Méry, Roselyne Bachelot avait entrepris de poser une question mettant clairement en cause le PS dans cette même affaire. Et, bien que cette question ait été posée par une femme souvent considérée comme féminine ${ }^{17}$, le registre de la controverse employé inscrivait bien davantage Roselyne Bachelot dans le modèle "polémique» masculin que dans le modèle "pragmatique empathique » féminin, pour reprendre les catégories proposées par Simone Bonnafous à propos des formes d'expression employées par certains ministres du gouvernement Jospin (Bonnafous, 2003) ${ }^{18}$.

Ce qui transparaît donc finalement de l'analyse de ces lapsus, c'est qu'ils ont pour principe une domination masculine dont le ressort essentiel n'est pas tant lié au sexe des individus mis en cause qu'au statut dont ils disposent ou à la manière dont ils en assurent l'exercice.

\section{Ordre du champ et inégalités de genre}

En fait, l'analyse des lapsus que nous avons menée atteste du fait que l'application involontaire du masculin et du féminin à l'égard des individus a pour

16. Le lapsus a été omis par le Journal officiel qui indique «Madame la députée » et non «Monsieur le député », expression qui a été relevée par le Service politique de Libération.

17. Pour Christine Bard, la carrière de Roselyne Bachelot s'est appuyée sur une performance de genre tournée vers la féminité (Bard, 2012).

18. Simone Bonnafous distingue en fait le modèle «polémique » adopté par Jean-Pierre Chevènement et Claude Allègre, le modèle «équilibré et pondéré» de L. Jospin et Dominique StraussKahn et le modèle «pragmatique empathique » mobilisé par Marie-George Buffet, Dominique Voynet, É. Guigou et M. Aubry (Bonnafous, 2003). 
ressort une valorisation différenciée des genres dans le champ politique, le féminin y disposant d'un «coefficient symbolique négatif» (Bourdieu, 1998, p. 100-101) relativement au masculin. Le lapsus commis à l'égard d'Yvon Robert, maire socialiste de Rouen, par l'écologiste Guillaume Grima, semble en témoigner (13).

(13) «Merci, madame le Maire de Rouen. Oh pardon, monsieur le Maire de Rouen...». A-t-il fait exprès? C'est en tout cas par ce lapsus que l'écologiste Guillaume Grima marque vendredi soir à sa manière le remplacement de Valérie Fourneyron par Yvon Robert.

(«Une majorité encore mal garée», Paris-Normandie Liberté Dimanche, 8 juillet 2012)

En utilisant la forme de «madame » dans le cadre d'un remerciement formulé à l'attention du maire, celui-ci avait en effet produit un lapsus qui entrait en résonance avec le manque de légitimité politique dont disposait alors Y. Robert et avec la position statutairement inférieure (selon l'ordre protocolaire) qu'il occupait vis-à-vis de V. Fourneyron. En effet, non seulement son accès à la fonction de maire n'avait pas été consacré par la "procédure électorale», qui veut que ce soit la tête de liste remportant les élections municipales qui devienne maire - procédure garante, dans une société où l'acte électoral est au cœur de l'accès aux fonctions politiques les plus prestigieuses, d'une forte légitimité démocratique 19 -, mais en outre, Y. Robert, qui avait été successivement suppléant de V. Fourneyron lors des élections législatives de 2007, puis son premier adjoint lorsqu'elle était devenue maire de Rouen, n'apparaissait pas aux yeux des acteurs locaux comme le «chef de file naturel du PS» rouennais ${ }^{20}$.

Mutatis mutandis, parce que les femmes peuvent être perçues d'abord et avant tout au travers de leur statut lorsqu'ils sont prestigieux, celles-ci peuvent en venir non seulement à disposer, du point de vue de celles et ceux qui parlent d'elles ou s'adressent à elles, des prérogatives liées à leurs fonctions, mais également à être perçues et catégorisées au masculin, autrement dit à bénéficier du privilège symbolique des dominants de la domination masculine. C'est dire, alors, l'enjeu qu'il peut y avoir pour certaines d'entre elles à se faire appeler au travers de titres sous une déclinaison masculine ${ }^{21}$ et l'importance des contro-

19. Y. Robert devient maire de Rouen avec la nomination de V. Fourneyron au poste de ministre des Sports, de la Jeunesse, de l'Éducation populaire et de la Vie associative. Le vert Jean-Michel Bérégovoy a fait savoir, lors du conseil municipal au cours duquel Y. Robert a été élu, que «pour beaucoup Yvon Robert a un manque de légitimité, non sur ses capacités, mais par rapport à l'acte démocratique que représente l'élection du maire» (Paris-Normandie, 8 juillet 2012).

20. Un journaliste rapporte ce propos d'Alexandre Canet, directeur de cabinet d'Y. Robert et secrétaire de la section locale du PS, pour qui V. Fourneyron est la « chef de file naturelle du parti socialiste à Rouen » (Paris-Normandie, 30 septembre 2012).

21. On peut alors comprendre la posture de Christine Lagarde qui, selon L'Express.fr, «tient au “le” [de Madame LE ministre] et n'est pas insensible à cette forme de révérence... » (L'Express.fr, 17 décembre 2009). 
verses que peut susciter la question de la féminisation des noms de métiers (voir à ce sujet le débat paru dans Travail, genre et sociétés : Collectif, 2000).

Mais il ne faudrait pas déduire de notre propos que les différences sexuelles n'ont aucun lien avec l'ordre du pouvoir politique. Le sexe constitue en effet un indice de la position dans la hiérarchie du pouvoir politique pour les acteurs du champ et joue à ce titre un rôle central dans la production des lapsus. On constate en effet que les lapsus d'adresse frappent davantage celles et ceux pour qui il n'y a pas congruence entre le genre associé à leur statut politique et celui associé à leur sexe (femmes dominantes, hommes dominés) que celles et ceux pour qui elle existe (femmes dominées, hommes dominants) (voir tableau 3).

Ainsi, on voit que lorsqu'un lapsus est commis par un acteur politique à l'égard d'une femme sans transgresser son identité sexuelle, dans $70 \%$ des cas, celle-ci possède un statut politique dominé alors que, lorsqu'un tel lapsus la transgresse (qu'une femme se fasse appeler «monsieur» ou qu'elle se fasse attribuer le prénom et/ou le nom d'un homme), celle-ci est dotée dans $66 \%$ des cas d'un statut dominant. À l'inverse, lorsqu'un lapsus est commis à l'égard d'un homme sans transgresser son identité sexuelle, dans $57 \%$ des cas, celui-ci possède un statut dominant alors que, lorsqu'un tel lapsus la transgresse, celui-ci est doté dans $60 \%$ des cas d'un statut dominé.

Tableau 3. Répartition des lapsus d'adresse commis par des acteurs du champ politique et mettant en cause au moins une personnalité politique selon l'existence d'une transgression sexuelle de leur identité (1871-2016)

\begin{tabular}{|c|c|c|c|c|c|c|}
\hline & $\begin{array}{l}F=> \\
F(2) \\
(e n \%)\end{array}$ & $\begin{array}{l}F=> \\
H(3) \\
\text { (en \%) }\end{array}$ & $\begin{array}{l}\text { Écart } \\
\text { (en } \\
\text { points) }\end{array}$ & $\begin{array}{l}H=> \\
H(2) \\
(\text { en \%) }\end{array}$ & $\begin{array}{l}H=> \\
F(3) \\
\text { (en \%) }\end{array}$ & $\begin{array}{c}\text { Écart } \\
\text { (en } \\
\text { points) }\end{array}$ \\
\hline $\begin{array}{l}\text { (A) Absence de mandat politique, } \\
\text { conseiller «territorial» (municipal, } \\
\text { départemental, etc.) }\end{array}$ & 57 & 16 & -41 & 31 & 20 & -11 \\
\hline $\begin{array}{l}\text { (B) Maire, président de collectivité } \\
\text { territoriale (communauté de com- } \\
\text { mune, conseil régional, etc.) }\end{array}$ & 4 & 16 & 11 & 10 & 20 & 10 \\
\hline (C) Député européen & 9 & 3 & -6 & 2 & 20 & 18 \\
\hline Statut dominé (1) $(A+B+C)$ & 70 & 34 & -35 & 43 & 60 & 17 \\
\hline (D) Député, sénateur & 22 & 38 & 16 & 31 & 20 & -11 \\
\hline (E) Ministre, secrétaire d’État & 9 & $\underline{19}$ & $\underline{10}$ & 13 & 10 & -3 \\
\hline $\begin{array}{l}\text { (F) Président de la République, chef } \\
\text { d’État, Premier ministre, président } \\
\text { du Conseil, président de chambre }\end{array}$ & - & 9 & 9 & 14 & 10 & -4 \\
\hline \multirow[t]{2}{*}{ Statut dominant $(1)(D+E+F)$} & 30 & 66 & 35 & 57 & 40 & -17 \\
\hline & $N=23$ & $N=32$ & & $N=213$ & $N=10$ & \\
\hline
\end{tabular}

Source : base de données personnelle. 
(1) : nous avons considéré que relevaient d'un lapsus concernant un individu disposant d'un statut dominé tous les cas où les individus dont le prénom et/ou le nom ou la forme de civilité étaient effacés n'avaient pas de mandat politique ou alors exerçaient un mandat local ou supra-national. Nous avons considéré que relevaient d'un lapsus concernant un individu disposant d'un statut dominant tous ceux où les individus dont le prénom et/ou le nom ou la forme de civilité étaient effacés exerçaient un mandat politique national ou disposaient d'un statut relevant de l'exécutif.

(2) $: F=>F(H=>H)$. À l'occasion d'un lapsus commis à l'égard d'une femme (d'un homme) et mettant en jeu au moins une personnalité politique, un acteur du champ politique a transgressé son adresse en lui attribuant une autre adresse féminine (masculine).

(3) : $F=>H(H=>F)$. À l'occasion d'un lapsus commis à l'égard d'une femme (un homme) et mettant en jeu au moins une personnalité politique, un acteur du champ politique a transgressé son adresse en lui attribuant une adresse de civilité inadaptée - monsieur (madame) - ou une adresse personnelle correspondant à celle d'un homme (d'une femme).

Lecture (données soulignées) : $19 \%$ des cas de lapsus de notre corpus (commis par des acteurs du champ politique et mettant en jeu au moins une personnalité politique) se traduisant par la transgression de l'adresse d'une femme en lui attribuant une adresse masculine le sont à l'égard de femmes disposant d'un statut de ministre ou de secrétaire d'État. C'est 10 points de plus que la part que représentent, parmi les cas de lapsus de notre corpus se traduisant par la transgression de l'adresse d'une femme en lui attribuant une autre adresse féminine, ceux commis à l'égard de femmes disposant d'un statut de ministre ou de secrétaire d'État.

\section{Pour conclure}

L'évolution des lapsus d'adresse commis par les acteurs du champ politique invite à penser que la domination masculine y persiste mais qu'elle est moins marquée depuis les lois sur la parité qu'elle n'avait pu l'être jusqu'alors. Par la féminisation du champ, la confusion entre le masculin et l'exercice du pouvoir politique s'est atténuée et a permis de voir se développer de manière significative la part des lapsus commis par les acteurs du champ politique au travers desquels le prénom et/ou le nom d'une personnalité féminine s'imposent. Si «la présence féminine en politique n'est pas acquise au point d'apparaître banale et ordinaire» (Pionchon, Derville, 2004, p. 96), l'analyse de notre corpus de lapsus invite à penser qu'elle s'y est banalisée et que le « féminin politique » s'est introduit dans les représentations des acteurs du champ.

Parallèlement, l'analyse des lapsus que nous avons menée atteste du fait que, dans son principe même, la domination masculine ne peut être réduite au triste constat des inégalités hommes-femmes. Dans le champ politique, elle est d'abord une affaire symbolique et est essentiellement liée à l'ordre du pouvoir. Le genre, « principe classificatoire» (Durkheim, Mauss, 1903), n’y est 
donc ni seulement ni parfaitement un élément qui recoupe l'identité sexuelle des individus : il apparaît plutôt comme un instrument de caractérisation de ces derniers qui se calque sur la valorisation associée à leur position statutaire.

Mais, parce que "le passé des relations sociales et ses sous-produits - matériel, idéologique, et autres - contraignent le présent des relations sociales» (Tilly, 1989, p. 29), il convient aussi de considérer que cette domination réside dans l'intériorisation, chez les agents de ce champ, du marquage genré des fonctions politiques et des comportements et attitudes par lesquels elles sont mises en œuvre. C'est bien alors en s'appuyant sur une réalité objective, sur une histoire politique intériorisée marquée du sceau de la masculinité, que le « dire » involontaire - mais aussi volontaire (Derville, Pionchon, 2005) de la «personnalité politique», et plus encore de la «fonction politique prestigieuse », s'opère d'abord et avant tout au masculin.

\section{Références}

ARNAUd Pierre, 1997, "Les ratés de la dénomination individuelle : typologie des lapsus par substitution de mots", dans C. Boisson et P. Thoinon éd., Autour de la dénomination, Lyon, Presses universitaires de Lyon, p. 307-331.

BARD Christine, 2012, "Performances de genre : images croisées de Michèle AlliotMarie [et] de Roselyne Bachelot», Histoire@Politique. Politique, culture, société, no 17, p. 69-86.

Bonnafous Simone, 2003, "“Femme politique” : une question de genre? », Réseaux, no 120 , p. 121-145.

Bourdieu Pierre, 1998, La domination masculine, Paris, Seuil.

- 1984, "Espace social et genèse des "classes" ", Actes de la recherche en sciences sociales, no52-53, p. 3-14.

- 1979, La distinction : critique sociale du jugement, Paris, Minuit.

ColleCtIF, 2000, "Autour du livre de Anne-Marie Houdebine La féminisation des noms de métiers", Travail, genre et sociétés, n03, p. 169-189.

Coniez Hugo, 2008, Écrire la démocratie : de la publicité des débats parlementaires, Paris, L'Harmattan.

Derville Grégory, Pionchon Sylvie, 2005, «La femme invisible : sur l'imaginaire du pouvoir politique», Mots. Les langages du politique, n ${ }^{0} 78$, p. 53-64.

DURKHEIM Émile, 2005 [1901], Les règles de la méthode sociologique, Paris, PUF.

DURKHEIM Émile, MAUSS Marcel, 1903, «De quelques formes primitives de classification: contribution à l'étude des représentations collectives ", L'Année sociologique, t. VI, p. 1-72 (repris dans M. Mauss, 1974, Euvres, t. II, Paris, Minuit, p. 13-89).

FREUd Sigmund, 2001 [1922], Psychopathologie de la vie quotidienne, S. Jankélévitch trad., Paris, Payot.

FROMKIN Victoria, 1971, «The non-anomalous nature of anomalous utterances », Language, vol.XLVII, nº1, p. 27-52 (repris dans V. Fromkin éd., 1973, Speech Errors as Linguistic Evidence, La Haye, Paris, Mouton, p. 215-242).

GoffMAn Erving, 1973, La mise en scène de la vie quotidienne. 1, La présentation de soi, Paris, Minuit. 
KERBRAT-ORECCHIONI Catherine éd., 2010, S'adresser à autrui : les formes nominales d'adresse en français, Chambéry, Université de Savoie, UFR Lettres, langues, sciences humaines, Laboratoire Langages, littératures, sociétés.

Marx Karl, Engels Friedrich, 1977 [1845], L'idéologie allemande, Paris, Éditions sociales. PIonchon Sylvie, DerviLLE Grégory, 2004, Les femmes et la politique, Grenoble, Presses universitaires de Grenoble.

SINEAU Mariette, 2011, Femmes et pouvoir sous la Ve République : de l'exclusion à l'entrée dans la course présidentielle, Paris, Presses de Sciences Po.

- 1988, Des femmes en politique, Paris, Economica.

TIlly Charles, 1989, "L'Histoire à venir », Politix. Revue des sciences sociales du politique, no 6, p. 25-32.

Weber Max, 1995 [1922], Économie et société. Tome I : Les catégories de la sociologie, Paris, Pocket.

\section{Résumé / Abstract / Compendio}

\section{Domination masculine et représentations sexuées des acteurs du champ politique : une approche par les lapsus d'adresse}

À partir d'une analyse des lapsus d'adresse commis par les acteurs du champ politique, l'objectif de notre article est de montrer que la confusion entre champ politique et masculinité qui caractérise les représentations de ces acteurs s'est atténuée depuis les lois sur la parité. Notre article vise également à montrer qu'elles restent marquées par la domination masculine.

Mots-clés : lapsus, domination masculine, champ politique, représentations sexuées, pouvoir

\section{Masculine domination and sexual representations of political field actors: an approach by mistitlings}

This article proposes an analysis of mistitlings committed by political field actors. It aims at showing that the confusion between the political field and masculinity which characterizes the representations of these actors has decreased since gender parity laws. But our article also aims to prove that their representations remain marked by masculine domination.

Keywords: slip of the tongue, masculine domination, political field, sexual representations, power

\section{Dominación masculina y representaciones del sexo de agentes del campo político: un análisis vía los lapsus de la denominación}

A partir de un análisis de los lapsus cometidos por agentes del campo político y que implican errores de la denominación, el objetivo de nuestro artículo es mostrar que la confusión entre el campo político y masculinidad que caracteriza las representaciones de estos agentes, se ha atenuado desde las leyes sobre la paridad. Nuestro artículo también pretende mostrar que siguen marcados por la dominación masculina.

Palabras claves: lapsus, dominación masculina, campo político, representaciones del sexo, poder 